Proceedings of the Edinburgh Mathematical Society (2002) 45, 617-630 (C)

DOI:10.1017/S0013091501000530 Printed in the United Kingdom

\title{
COMBINATORIAL TECHNIQUES FOR DETERMINING RANK AND IDEMPOTENT RANK OF CERTAIN FINITE SEMIGROUPS
}

\author{
INESSA LEVI AND STEVE SEIF \\ Department of Mathematics, University of Louisville, Louisville, KY 40292, USA \\ (inessa.levi@louisville.edu; swseif01@louisville.edu)
}

(Received 23 April 2001)

\begin{abstract}
Let $\tau$ be a partition of the positive integer $n$. A partition of the set $\{1,2, \ldots, n\}$ is said to be of type $\tau$ if the sizes of its classes form the partition $\tau$ of $n$. It is known that the semigroup $S(\tau)$, generated by all the transformations with kernels of type $\tau$, is idempotent generated. When $\tau$ has a unique non-singleton class of size $d$, the difficult Middle Levels Conjecture of combinatorics obstructs the application of known techniques for determining the rank and idempotent rank of $S(\tau)$. We further develop existing techniques, associating with a subset $U$ of the set of all idempotents of $S(\tau)$ with kernels of type $\tau$ a directed graph $D(U)$; the directed graph $D(U)$ is strongly connected if and only if $U$ is a generating set for $S(\tau)$, a result which leads to a proof if the fact that the rank and the idempotent rank of $S(\tau)$ are both equal to
\end{abstract}

$$
\max \left\{\left(\begin{array}{l}
n \\
d
\end{array}\right),\left(\begin{array}{c}
n \\
d+1
\end{array}\right)\right\}
$$

Keywords: semigroup; digraph; perfect matching; Middle Levels Conjecture; idempotent rank

AMS 2000 Mathematics subject classification: Primary 20M20; 05A18; 05A17; 05C20

\section{Introduction}

The rank of a finite semigroup $S$ is the size of a minimal set of generators of $S$. An element $e$ contained in $S$ is idempotent if $e^{2}=e$. If $S$ is generated by its idempotents, then the size of a minimal generating set of idempotents is referred to as the idempotent rank of $S$. In the present paper, combinatorial techniques are developed to investigate idempotent-generated semigroups of transformations of finite sets with respect to their rank and idempotent rank.

For a positive integer $n$, let $X_{n}=\{1, \ldots, n\}$ and let $T_{n}$ be the semigroup of all transformations from $X_{n}$ to itself. With a transformation $f \in T_{n}$ are associated its range, $\operatorname{ran}(f)=f\left(X_{n}\right)$, its height, $|\operatorname{ran}(f)|$, and its kernel, $\operatorname{ker}(f)=\left\{(a, b) \in X_{n} \times X_{n}\right.$ : $f(a)=f(b)\}$.

For a positive integer $r \leqslant n-1$, let $K(n, r)$ be the subsemigroup of $T_{n}$ consisting of all transformations of height at most $r$. Howie shows in [5] that $K(n, r)$ is idempotent generated, and in $[\mathbf{6}]$ he establishes a one-to-one correspondence between minimal generating 
sets of $K(n, n-1)$ and the set of strongly connected tournaments, a correspondence we describe in Remark 3.4 below.

Recall that the Stirling number $S(n, r)$ is the number of partitions of $X_{n}$ of weight $r$. A partition $\pi$ of $X_{n}$ and an $r$-set $A$ contained in $X_{n}$ are said to be orthogonal if every class of $\pi$ contains exactly one element of $A$. An orthogonally labelled list $A_{1} \pi_{1} \ldots A_{\left(\begin{array}{c}n \\ r\end{array}\right)} \pi_{\left(\begin{array}{c}n \\ r\end{array}\right)}$ is an alternating sequence of distinct $r$-sets $A_{1}, \ldots, A_{\left(\begin{array}{c}n \\ r\end{array}\right)}$ and distinct partitions $\pi_{1}, \ldots, \pi_{\left(\begin{array}{c}n \\ r\end{array}\right)}$, such that for $i=1, \ldots,\left(\begin{array}{l}n \\ r\end{array}\right)-1, \pi_{i}$ is orthogonal to $A_{i}$ and $A_{i+1}$, and $\pi_{\left(\begin{array}{c}n \\ r\end{array}\right)}$ is orthogonal to $A_{\left(\begin{array}{c}n \\ r\end{array}\right)}$ and $A_{1}$. In [7], Howie and McFadden prove that for every $n>r$, there exist orthogonally labelled lists - a result leading to their main theorem, which states that the idempotent rank and the rank of $K(n, r)$ are equal to $S(n, r)$. We describe their technique in more detail in Remark 3.9 below.

A partition of the set $X_{n}$ has type $\tau=d_{1}{ }^{t_{1}} d_{2}{ }^{t_{2}} \ldots d_{k}{ }^{t_{k}}$ if it has $t_{i}$ classes of size $d_{i}$, where $d_{1}>d_{2}>\cdots>d_{k}, i=1,2, \ldots, k$, and $n=\sum_{i=1}^{k} d_{i} t_{i}$. The number $r=\sum_{i=1}^{k} t_{i}$ of classes of $\tau$ is the weight of $\tau$. For a given partition type $\tau$ on $X_{n}$, let $S(\tau)$ be the semigroup generated by all the transformations whose kernels have type $\tau$. The semigroup $S(\tau)$ is invariant under the conjugation by the permutations of $X_{n}$, and so it is idempotent generated [9]. An orthogonally $\tau$-labelled list is an orthogonally labelled list $A_{1} \pi_{1} \ldots A_{\left(\begin{array}{c}n \\ r\end{array}\right)} \pi_{\left(\begin{array}{c}n \\ r\end{array}\right)}$ as above, in which all the partitions $\pi_{1}, \ldots, \pi_{\left(\begin{array}{c}n \\ r\end{array}\right)}$ are of type $\tau$.

The existence of orthogonally $\tau$-labelled lists leads to a determination of the rank and idempotent rank of $S(\tau)$ (see $[\mathbf{8}, \mathbf{1 0}]$ ); however, it is not always easy to prove that orthogonally $\tau$-labelled lists exist, if indeed they do exist. In fact, for $\tau=r 1^{r-1}$, the existence of an orthogonally $r 1^{r-1}$-labelled list is equivalent to the validity of the difficult Middle Levels Conjecture for $r$, as we show in Remark 3.10. In order to determine the rank and idempotent rank of $S\left(d 1^{r-1}\right)$ semigroups without proving the Middle Levels Conjecture, we further develop the techniques of $[\mathbf{6}]$ and $[\mathbf{7}]$, by associating a certain directed graph with a subset of idempotents of a semigroup. Our techniques can be applied to a broad range of semigroups of transformations of finite sets, including certain semigroups of endomorphisms [15]. Here the techniques are used to determine the rank and idempotent rank of $S\left(d 1^{r-1}\right)$ semigroups.

Theorem 1.1. Let $\tau$ be a partition type $d 1^{r-1}$, with $d \geqslant 2$ and $r \geqslant 2$. With $n=$ $d+r-1$, the following conditions hold.

(1) If $r \geqslant d$, then both the rank and the idempotent rank of $S(\tau)$ are $\left(\begin{array}{c}n \\ r-1\end{array}\right)$.

(2) If $d>r$, then both the rank and the idempotent rank of $S(\tau)$ are $\left(\begin{array}{l}n \\ r\end{array}\right)$.

In particular, the rank and the idempotent rank of $S(\tau)$ are equal to

$$
\max \left\{\left(\begin{array}{c}
n \\
r-1
\end{array}\right),\left(\begin{array}{l}
n \\
r
\end{array}\right)\right\} .
$$

The work here is part of a recently completed program $[\mathbf{1 0}-\mathbf{1 2}]$ aimed at determining the rank and the idempotent rank of all $S(\tau)$ semigroups. 


\section{Background on semigroups of transformations and their rank and idempotent rank}

Notation and elementary results concerning semigroups of transformation and their rank and idempotent rank are provided below. Let $\mathcal{A}_{n, r}$ be the set of all $r$-sets of $X_{n}$. The statements in the next observation will be used without comment in the sequel.

\section{Lemma 2.1.}

(1) Let $f$ and $g$ be transformations of $X_{n}$.

If $|\operatorname{ran}(f)|=|\operatorname{ran}(g)|$, then the following are equivalent:
(a) $|\operatorname{ran}(f g)|=|\operatorname{ran}(f)|$;
(b) $\operatorname{ran}(f g)=\operatorname{ran}(f)$;
(c) $\operatorname{ran}(g)$ is orthogonal to $\operatorname{ker}(f)$; and
(d) $\operatorname{ker}(f g)=\operatorname{ker}(g)$.

(2) If $A$ is an $r$-subset of $X_{n}$ and $\pi$ is a partition of $X_{n}$ of weight $r$, then there exists a transformation $f$ of $T_{n}$ such that $\operatorname{ran}(f)=A$ and $\operatorname{ker}(f)=\pi$.

An idempotent transformation $e$ of $X_{n}$ is the identity transformation on its range $\operatorname{ran}(e)$; that is, for all $x \in \operatorname{ran}(e)$, we have $e(x)=x$. There is a one-to-one correspondence between idempotents of height $r$ and pairs $(\pi, A)$, where $\pi$ is a weight $r$ partition of $X_{n}$ and $A$ is an $r$-set orthogonal to $\pi$. We often identify an idempotent with its associated pair $(\pi, A)$, where $\pi=\operatorname{ker}(e)$ and $A=\operatorname{ran}(e)$.

A transformation $f$ of $X_{n}$ is said to be a group element if it belongs to a subgroup of the semigroup $T_{n}$. In fact, $f$ is a group element if and only if the range $\operatorname{ran}(f)$ is orthogonal to the partition $\operatorname{ker}(f)$. If $f$ is a group element, by the finiteness of $X_{n}$, there exists a positive integer $m$ such that $f^{m}$ is an idempotent whose kernel and range coincide with those of $f$; that is, $f^{m}=(\operatorname{ker}(f), \operatorname{ran}(f))$.

That $S(\tau)$ semigroups are idempotent generated has been observed in [9]. Sufficient conditions for a set to be a generating set of an $S(\tau)$ semigroup are provided in the next easy lemma.

Lemma 2.2. Let $G$ be a subset of the semigroup $S(\tau)$. If for every partition $\alpha$ of type $\tau$ and every set $A$ orthogonal to $\alpha$ there exists an $f$ in $\langle G\rangle$ such that $\operatorname{ker}(f)=\alpha$ and $\operatorname{ran}(f)=A$, then $G$ is a generating set for $S(\tau)$.

Proof. Because $S(\tau)$ is idempotent generated, it suffices to show that an arbitrary idempotent of height $r$ in $S(\tau)$ is contained in $\langle G\rangle$. Let $e$ be such an idempotent with $\operatorname{ker}(e)=\alpha$ and $\operatorname{ran}(e)=A$. Since $\alpha$ is orthogonal to $A$ there exists an $f \in\langle G\rangle$, with $\operatorname{ker}(f)=\alpha$ and $\operatorname{ran}(f)=A$. Since $\alpha$ and $A$ are orthogonal, the transformation $f$ is a group element; thus, there exists a positive integer $m$ such that $f^{m}$ is an idempotent. Since any idempotent is uniquely determined by its range and partition, we have $f^{m}=e$. 


\section{1. $S(\tau)$ semigroups, $\tau=d 1^{r-1}$}

For the remainder of the paper, unless stated otherwise, we consider only $S(\tau)$ semigroups, where $\tau$ is $d 1^{r-1}$ with $d \geqslant 2$ and $r \geqslant 2$; we set $n$ equal to $d+r-1$. Let $T(\tau)$ be the set of all partitions of type $\tau$ and recall that $\mathcal{A}_{n, r}$ is the set of all $r$-sets of $X_{n}$. In the next lemma, we collect a number of observations concerning $S(\tau)$ semigroups.

Lemma 2.3. Let $\tau$ be $d 1^{r-1}$.

(1) There are $\left(\begin{array}{c}n \\ d\end{array}\right)=\left(\begin{array}{c}n \\ r-1\end{array}\right)$ distinct partitions in $T(\tau)$. Thus the set $\{\operatorname{ker}(f): f \in$ $S(\tau)$, height $(f)=r\}$ has $\left(\begin{array}{c}n \\ r-1\end{array}\right)$ elements.

(2) The set $\{\operatorname{ran}(f): f \in S(\tau)$, height $(f)=r\}$ has $\left(\begin{array}{l}n \\ r\end{array}\right)$ elements.

(3) Let $A$ and $B$ be $r$-sets contained in $\mathcal{A}_{n, r}$ and let $\alpha$ be a partition of type $d 1^{r-1}$. Then the following hold:

(a) the set $A$ is orthogonal to $r$ distinct partitions of type $d 1^{r-1}$; the partition $\alpha$ is orthogonal to $d$ distinct sets contained in $\mathcal{A}_{n, r}$;

(b) there exists a partition of type $d 1^{r-1}$ which is simultaneously orthogonal to $A$ and $B$ if and only if $|A \cap B|=r-1$; and

(c) if $|A \cap B|=r-1$, there is a unique partition simultaneously orthogonal to $A$ and $B$, a partition which we denote by $\pi(A, B)$.

(4) There is a bijection from $T(\tau)$ to $\mathcal{A}_{n, r-1}$, under which a partition $\alpha$ of type $\tau$ is mapped to the $(r-1)$-set of singleton classes of $\alpha$.

The rank of $S(\tau)$ is at least as large as $\max \left\{\left(\begin{array}{c}n \\ r-1\end{array}\right),\left(\begin{array}{l}n \\ r\end{array}\right)\right\}$. Indeed, for a set $A \in \mathcal{A}_{n, r}$ and a partition $\alpha$ of type $\tau$, there exists $f \in S(\tau)$ such that $\operatorname{ran}(f)=A, \operatorname{ker}(f)=\alpha$. If $G$ is a generating set for $S(\tau)$, there exist transformations $g_{1}, g_{2}, \ldots, g_{k} \in G$ such that $f=$ $g_{1} g_{2} \ldots g_{k}$. Since the height of each of $g_{1}, g_{2}, \ldots, g_{k}$ must be $r$, it follows that $\operatorname{ran}\left(g_{1}\right)=$ $\operatorname{ran}(f)=A$ and $\operatorname{ker}\left(g_{k}\right)=\operatorname{ker}(f)=\alpha$. Therefore, for each $\alpha \in T(\tau)$, the generating set $G$ contains at least one transformation $g$ with $\operatorname{ker}(g)=\alpha$, which implies that $|G| \geqslant\left(\begin{array}{c}n \\ r-1\end{array}\right)$. Moreover, for any $A \in \mathcal{A}_{n, r}$, the set $G$ contains at least one transformation $g^{\prime}$ with $\operatorname{ran}\left(g^{\prime}\right)=A$; hence, $|G| \geqslant\left(\begin{array}{l}n \\ r\end{array}\right)$. We extend these observations below, providing necessary conditions for a subset $G$ of $S(\tau)$ to serve as a minimal generating set.

Proposition 2.4. For a minimal generating set $G$ of $S(\tau)$, we have:

(1) $|G| \geqslant \max \left\{\left(\begin{array}{l}n \\ r\end{array}\right),\left(\begin{array}{c}n \\ r-1\end{array}\right)\right\}$;

(2) if the idempotent rank of $S(\tau)$ is equal to $\max \left\{\left(\begin{array}{l}n \\ r\end{array}\right),\left(\begin{array}{c}n \\ r-1\end{array}\right)\right\}$, then the rank of $S(\tau)$ is equal to its idempotent rank;

(3) for each $\alpha \in T(\tau)$ there is at least one $g \in G$ with $\operatorname{ker}(g)=\alpha$; if $|G| \leqslant\left(\begin{array}{c}n \\ r-1\end{array}\right)$, then such $g$ is unique; 
(4) for each $A \in \mathcal{A}_{n, r}$ there is at least one $g \in G$ with $\operatorname{ran}(g)=A$; if $|G| \leqslant\left(\begin{array}{l}n \\ r\end{array}\right)$, then such a $g$ is unique;

(5) $|\operatorname{ran}(g)|=r$ for each $g \in G$.

\subsection{Duality of idempotents for $S(\tau)$}

The goal here is to reduce the proof of Theorem 1.1 to the case when $d \leqslant r$. We do so by using an unusual duality property satisfied by the idempotents of $S(\tau)$, when $\tau$ is $d 1^{r-1}$.

Recall that every idempotent $e$ may be identified with an ordered pair $(\operatorname{ker}(e), \operatorname{ran}(e))$. If the kernel of $e$ is a partition of type $d 1^{r-1}$, then $e$ can be also identified with another ordered pair, the pair $(a, \operatorname{ran}(e))$, where $a$ is the unique element contained in the intersection of $\operatorname{ran}(e)$ and the unique non-singleton class of $\operatorname{ker}(e)$; we write $e=(a, A)$, where $A=\operatorname{ran}(e)$. For a subset $B$ of $X_{n}$, the complement of $B$ in $X_{n}$ is denoted $B^{\mathrm{C}}$.

With a height $r$ idempotent $e=(a, A)$ contained in $T_{n}$ with kernel of type $\tau=d 1^{r-1}$, we associate its dual idempotent $\bar{e}=\left(a, A^{\mathrm{C}} \cup\{a\}\right)$. Note that $\bar{e}$ is a transformation in $T_{n}$ whose kernel is of type $r 1^{d-1}$, the range is $A^{\mathrm{C}} \cup\{a\}$, and the height is $d$. Moreover, we have that $\overline{\bar{e}}=e$. For $\tau=d 1^{r-1}$, let $\bar{\tau}=r 1^{d-1}$; observe also that $\overline{\bar{\tau}}$ is $\tau$.

\section{Proposition 2.5.}

(1) If $G=\left\{e_{1}, e_{2}, \ldots, e_{k}\right\}$ is a minimal generating set for $S(\tau)$, then $\bar{G}=\left\{\overline{e_{1}}, \overline{e_{2}} \ldots, \overline{e_{k}}\right\}$ is a minimal generating set for $S(\bar{\tau})$.

(2) If the idempotent rank of $S(\tau)$ is $\left(\begin{array}{l}n \\ r\end{array}\right)$, then the idempotent rank of $S(\bar{\tau})$ is $\left(\begin{array}{l}n \\ d\end{array}\right)$.

Proof. Let $e$ and $f$ be idempotents whose kernels are of type $\tau=d 1^{r-1}$. If $\operatorname{ran}(e)$ is orthogonal to $\operatorname{ker}(f)$, then from the definition of dual idempotents, it follows directly that $\operatorname{ran}(\bar{f})$ and $\operatorname{ker}(\bar{e})$ are also orthogonal. In particular, for a product of idempotents $e_{1} \ldots e_{m}$, each with kernel of type $\tau$, the height of $e_{1} \ldots e_{m}$ is $r$ if and only if the height of $\bar{e}_{m} \ldots \bar{e}_{1}$ is $d$.

Let $\alpha$ be a partition of $X_{n}$ of type $\bar{\tau}$, and let $\alpha$ be orthogonal to $A$. In view of Lemma 2.2, to prove that $\bar{G}$ is a generating set for $S(\bar{\tau})$, we only need to show that there exists a transformation $f \in\langle\bar{G}\rangle$ with $\operatorname{ran}(f)=A$ and $\operatorname{ker}(f)=\alpha$.

Let $B$ be the unique non-singleton class of the partition $\alpha$, and let $a$ be the unique element contained in the intersection of $A$ and $B$. Let $e=(a, B)$, the idempotent in $S(\tau)$ with range $B$ whose kernel of type $\tau$ has the unique non-singleton class $B^{\mathrm{C}} \cup\{a\}=A$. Since $G$ is a generating set for $S(\tau)$, there exist idempotents $e_{1}, \ldots, e_{m}$ such that $e=$ $e_{1} e_{2} \ldots e_{m}$, where $e_{i}=\left(a_{i}, A_{i}\right)$, for $i=1,2, \ldots m$. Let $f=\bar{e}_{m} \bar{e}_{m-1} \ldots \bar{e}_{1} \in\langle\bar{G}\rangle$. From the first paragraph, it follows that $f$ has range $A$ and kernel $\alpha$.

Corollary 2.6. Let $\tau=d 1^{r-1}$. If the rank and the idempotent rank of $S(\tau)$ are equal to $\left(\begin{array}{c}n \\ r-1\end{array}\right)$, then the rank and the idempotent rank of $S(\bar{\tau})$ are equal to $\left(\begin{array}{c}n \\ r-1\end{array}\right)=\left(\begin{array}{l}n \\ d\end{array}\right)$.

Notice that as a consequence of Corollary 2.6 and Proposition 2.4 (2), to prove Theorem 1.1 it suffices to prove that when $r \geqslant d$, the idempotent rank of $S(\tau)$ is $\left(\begin{array}{c}n \\ r-1\end{array}\right)$. 


\section{Distributions and generating sets}

The sets $T(\tau)$ and $\mathcal{A}_{n, r}$ are interconnected by partial functions, as defined below, via the orthogonality property.

Definition 3.1. A partial distribution is a partial surjective function

$$
\delta: T(\tau) \rightarrow \mathcal{A}_{n, r}
$$

satisfying the following condition: if $\pi$ is in the domain of $\delta$, then $\delta(\pi)$ is orthogonal to $\pi$. A distribution is a partial distribution whose domain is all of $T(\tau)$.

For a partial distribution $\delta$, let $\operatorname{dom}(\delta)$ denote the domain of $\delta$. Observe that each partial distribution $\delta$ determines a set of idempotents $I=\{(\pi, \delta(\pi)): \pi \in \operatorname{dom}(\delta)\}$, with distinct kernels and with the property that $\{\operatorname{ran}(e): e \in I\}$ is equal to $\mathcal{A}_{n, r}$; conversely, a set of idempotents $I$, with distinct kernels whose images comprise $\mathcal{A}_{n, r}$, determines a partial distribution. With a partial distribution (equivalently, with a set of idempotents satisfying the two properties above), we associate, in Definition 3.2 below, a certain directed graph $D(\delta)$. Necessary definitions from graph theory are provided.

A simple graph $U=(V, E)$ consists of a set of vertices $V$, together with a set $E$ of two element subsets of $V$ called the edges of $U$. A directed graph (or a digraph) $D=(V, E)$ consists of a set $V$ of vertices together with a set $E$ of directed pairs of distinct vertices, called directed edges. With every digraph $D$ one may associate an underlying simple graph which has the same vertices as $D$, and whose undirected edges consist of pairs of vertices which have a directed edge in $D$.

For a simple graph $U=(V, E)$, a walk is the alternating sequence $v_{1}, e_{1}, v_{2}, e_{2}, \ldots$, $e_{k-1}, v_{k}$, where $v_{1}, v_{2}, \ldots, v_{k}$ are vertices, and $e_{1}, e_{2}, \ldots, e_{k-1}$ are edges with $e_{i}=$ $\left\{v_{i}, v_{i+1}\right\}$. A path is a walk through distinct vertices. A path $v_{1}, e_{1}, v_{2}, e_{2}, \ldots, e_{k-1}, v_{k}$ is often written as $v_{1} v_{2} \ldots v_{k}$; the vertices $v_{1}$ and $v_{k}$ are said to be joined by the path $v_{1} v_{2} \ldots v_{k}$. If $k \geqslant 4$, the vertices $v_{1}, v_{2}, \ldots, v_{k-1}$ are distinct, and $v_{k}=v_{1}$, then the walk $v_{1} v_{2} \ldots v_{k}$ is referred to as a cycle. The above definitions are extended to a digraph by replacing 'edges' with 'directed edges'.

A simple graph is said to be connected if every pair of its vertices is joined by a path. A digraph is said to be connected if its underlying simple graph with undirected edges is connected. A digraph is strongly connected if for every pair of vertices $u$ and $v$, there exists a directed path beginning with $u$ and ending with $v$.

Definition 3.2. A partial distribution $\delta$ determines a directed graph $D(\delta)$ whose vertex set is $\mathcal{A}_{n, r}$, and whose edge set is defined as follows: for $A, B \in \mathcal{A}_{n, r}, A B$ is an edge of $D(\delta)$ if there exists a partition $\alpha \in T(\tau)$ such that $B=\delta(\alpha)$ and $\alpha$ is orthogonal to $A$.

Recall that for $\tau=d 1^{r-1}$, the idempotent rank of $S(\tau)$ is at least as large as $\left(\begin{array}{c}n \\ r-1\end{array}\right)$.

Proposition 3.3. Let $\tau=d 1^{r-1}$ with $d \leqslant r$. Then the idempotent rank of $S(\tau)$ is equal to $\left(\begin{array}{c}n \\ r-1\end{array}\right)$ if and only if there exists a partial distribution $\delta$ whose directed graph $D(\delta)$ is strongly connected. 
Proof. Suppose that the idempotent rank of $S(\tau)$ is $\left(\begin{array}{c}n \\ r-1\end{array}\right)$, and $G$ is a generating set of $S(\tau)$ consisting of $\left(\begin{array}{c}n \\ r-1\end{array}\right)$ idempotents. The range of each idempotent $e$ in $G$ is orthogonal to its kernel. Thus, the set $G$ gives rise to a function $\delta$ with domain $\{\operatorname{ker}(e): e \in G\}$, defined by $\delta(\alpha)=\operatorname{ran}(e)$, where $e$ is the idempotent in $G$ with $\operatorname{ker}(e)=\alpha$. Note that by Proposition 2.4(3), we have that $e$ is the only element of $G$ with kernel equal to $\alpha$. Thus, $\delta: T(\tau) \rightarrow \mathcal{A}_{n, r}$ is well defined as a partial function. Because $G$ is a generating set for $S(\tau)$, it follows from Proposition 2.4 (4) that the range of $\delta$ is $\mathcal{A}_{n, r}$. Therefore, $\delta$ is a partial distribution (in fact, a distribution).

We prove that the associated digraph $D(\delta)$, as given in Definition 3.2, is strongly connected. For $r$-sets $A$ and $B$ contained in $\mathcal{A}_{n, r}$, the surjectivity of $\delta$ guarantees the existence of partitions $\alpha$ and $\beta$ in the domain of $\delta$ such that $\delta(\alpha)=A$ and $\delta(\beta)=B$. By Lemma $2.1(2)$, there exists $f \in S(\tau)$ such that $\operatorname{ker}(f)=\alpha$ and $\operatorname{ran}(f)=B$. Since $G$ is a generating set of $S(\tau)$, there exist $e_{1}, e_{2}, \ldots, e_{k} \in G$ such that $f=e_{1} e_{2} \ldots e_{k}$. For $i=1,2, \ldots, k$, let $\operatorname{ker}\left(e_{i}\right)=\alpha_{i}$ and $\operatorname{ran}\left(e_{i}\right)=A_{i}$. By the definition of the partial distribution $\delta$, we have $\delta\left(\alpha_{i}\right)=A_{i}$. By Lemma 2.1(1) it follows that $\alpha_{i-1}$ is orthogonal to $A_{i}(i=2, \ldots, k)$, and by the definition of $D(\delta)$, there exists a directed edge from $A_{i}$ to $A_{i-1}$. Because $G$ is a minimal generating set, by Proposition 2.4 we must have that $\alpha_{k}=\operatorname{ker}\left(e_{k}\right)=\operatorname{ker}(f)=\alpha$. Since $\delta(\alpha)=A$, we also have $A_{k}=A$. Because we have $A_{1}=\operatorname{ran}\left(e_{1}\right)=\operatorname{ran}(f)=B$, we have shown that there exists a directed path from $A$ to $B$, and so $D(\delta)$ is strongly connected.

Conversely, suppose $\delta$ is a partial distribution whose associated digraph $D(\delta)$ is strongly connected. To show that $\delta$ determines a generating set of idempotents of the semigroup $S(\tau)$, define a distribution $\delta_{1}$ by extending the domain of $\delta$ to $T(\tau)$ as follows. For each partition $\alpha$ of type $\tau$ not contained in the domain of $\delta$, choose an $r$-set $A$ orthogonal to $\alpha$. Let $\delta_{1}$ coincide with $\delta$ on the domain of $\delta$, and for each $\alpha$ not in the domain of $\delta$, let $\delta_{1}(\alpha)=A$, where $A$ is chosen as above. Since $D(\delta)$ is strongly connected, the digraph $D\left(\delta_{1}\right)$ is also strongly connected.

Let $G$ be equal to $\left\{\left(\alpha, \delta_{1}(\alpha)\right): \alpha \in T(\tau)\right\}$; thus, $G$ consists of $\left(\begin{array}{c}n \\ r-1\end{array}\right)$ idempotents, all having distinct kernels. Let $f=(\alpha, A)$ be an arbitrary idempotent of height $r$, with kernel $\alpha$ of type $\tau$. We complete the proof by showing that $f \in\langle G\rangle$. Let $e=(\alpha, B)$ be the unique idempotent in $G$ with kernel $\alpha$. Since $D(\delta)$ is strongly connected, there exists a directed path $B=B_{0} B_{1} \ldots B_{m}=A$ in $D(\delta)$. For each directed edge $B_{i-1} B_{i}(i=1, \ldots, m)$, there exists a partition $\alpha_{i}$ such that $\delta\left(\alpha_{i}\right)=B_{i}$, where $\alpha_{i}$ is simultaneously orthogonal to $B_{i}$ and $B_{i-1}$. Let $e_{i}=\left(\alpha_{i}, B_{i}\right)$, for $i=1, \ldots, m$. The product $e_{m} e_{m-1} \ldots e_{1} e$ has kernel $\alpha$ and range $A$. Because $\alpha$ is orthogonal to $A$, we have that $e_{m} e_{m-1} \ldots e_{1} e$ is a group element; thus, there exists a positive integer $k$ such that $\left(e_{m} e_{m-1} \ldots e_{1} e\right)^{k}$ is the idempotent $(\alpha, A)=f$.

We have proven that the idempotent rank is no greater than $\left(\begin{array}{c}n \\ r-1\end{array}\right)$. In view of Lemma $2.4(2)$, it follows that the idempotent rank of $S(\tau)$ is $\left(\begin{array}{c}n \\ r-1\end{array}\right)$.

From the proof of Proposition 3.3 it is clear that with 'distribution' in place of 'partial distribution', the statement of Proposition 3.3 remains valid. The notion of partial distribution provides useful flexibility for the final stage of the proof of Theorem 1.1. 
Remark 3.4. Distributions and their associated directed graphs generalize the method of [6], for producing minimal idempotent generating sets for $K(n, n-1)$.

Indeed, recall that $K(n, n-1)$ is generated by its idempotents of height $n-1$. For $f \in K(n, n-1)$, if $f$ has height $n-1$, then $\operatorname{ker}(f)$ is of type $21^{n-2}$. It follows that $S\left(21^{n-2}\right)$ is equal to $K(n, n-1)$.

A tournament on $n$ vertices is a directed graph in which every pair of vertices is connected by exactly one directed edge. Let $T$ be a tournament on the $n$ vertices $X_{n}$. To establish a connection between minimal generating sets for $K(n, n-1)$ and strongly connected tournaments, in [6], the author associates with each vertex $k \in X_{n}$ the $(n-1)$ set $X_{n}-\{k\}$, and to each directed edge $j k\left(j, k \in X_{n}, j \neq k\right)$ the height $n-1$ idempotent with range $X_{n}-\{j\}$, and a kernel with unique non-singleton class is $\{j, k\}$. Thus, a tournament $T$ with vertex set $X_{n}$ determines a set $G$ of $\left(\begin{array}{l}n \\ 2\end{array}\right)$ idempotents with distinct kernels.

It is proven in $[\mathbf{6}]$ that if the tournament $T$ is strongly connected, then $G$ is a generating set for $K(n, n-1)$, and, conversely, any generating set consisting of $\left(\begin{array}{l}n \\ 2\end{array}\right)$ idempotents determines a strongly connected tournament on $n$ vertices.

As observed in Lemma 2.3 (3) (a), a partition $\pi$ of type $21^{n-2}$ is orthogonal to exactly two $(n-1)$-sets. In particular, for a distribution $\delta: T\left(21^{n-2}\right) \rightarrow \mathcal{A}_{n, n-1}$, the associated digraph $D(\delta)$ is a tournament. Because $\delta$ is a distribution, it has a domain consisting of all $\left(\begin{array}{l}n \\ 2\end{array}\right)$ partitions of $T(\tau)$. Hence, $\delta$ determines a set $G$ of $\left(\begin{array}{l}n \\ 2\end{array}\right)$ idempotents. By the proof of Proposition 3.3, $G$ is a generating set if and only if $D(\delta)$ is strongly connected. Thus, Proposition 3.3 leads to the result of $[6]$.

It is not difficult to construct tournaments which are not strongly connected. Thus, the directed graph associated with a distribution is not necessarily strongly connected.

\subsection{Distributions for $\tau=r 1^{r-1}$ - the Middle Levels Conjecture}

In this section the Middle Levels Conjecture and its connection with the determination of the rank and idempotent rank of $S\left(r 1^{r-1}\right)$ semigroups are described, and Theorem 1.1 is proved for $\tau=r 1^{r-1}$.

A simple graph $(V, E)$ is Hamiltonian if there exists a cycle of $(V, E)$ passing through all its vertices. Consider the graph $\mathcal{B}(r)$ whose set of vertices is the union of two sets, $\mathcal{A}_{2 r-1, r}$ and $\mathcal{A}_{2 r-1, r-1}$, such that an $r$-set and an $(r-1)$-set are adjacent when the smaller set is a subset of the larger set. The Middle Levels Conjecture for $r$, usually attributed to Paul Erdos $[\mathbf{3}, \mathbf{1 4}]$, states that $\mathcal{B}(r)$ is a Hamiltonian graph.

A perfect matching of $\mathcal{B}(r)$ is a set of $\left(\begin{array}{c}2 r-1 \\ r\end{array}\right)$ edges, no two of which have a vertex in common. It is non-trivial to show that $\mathcal{B}(r)$ has perfect matchings [1]. It is thought that developing a better understanding of perfect matchings of $\mathcal{B}(r)$ may lead to a proof of the validity of the Middle Levels Conjecture [4].

For a partition $\alpha$ of type $r 1^{r-1}$, let $B(\alpha)$ be the $(r-1)$-set of singleton classes of $\alpha$. Every distribution $\delta$, associated with the partition type $r 1^{r-1}$, gives rise to a perfect matching $M_{\delta}$ of $\mathcal{B}(r)$, where $M_{\delta}$ consists of the edges $\left\{B(\alpha) \delta(\alpha): \alpha \in T\left(r 1^{r-1}\right)\right\}$. Conversely, a perfect matching $M$ of $\mathcal{B}(r)$ determines a distribution $\delta_{M}$ for the type $r 1^{r-1}$, as is quite easy to verify. 
Note that the partition type $r 1^{r-1}$ is special in another way; this is the only partition type of the form $\tau=d 1^{r-1}$, for which the number of partitions of $X_{n}$ of type $\tau$ equals the number of the $r$-subsets of $X_{n}$. Thus, every partial distribution for $\tau=r 1^{r-1}$ is a distribution, and so the partial distributions for the type $r 1^{r-1}$ are in one-to-one correspondence with the perfect matchings of $\mathcal{B}(r)$. In view of the fact that perfect matchings exist for $\mathcal{B}(r)[\mathbf{1}]$, we have the following lemma.

Lemma 3.5. For $\tau=r 1^{r-1}$, there exists a distribution $\delta: T(\tau) \rightarrow \mathcal{A}_{2 r-1, r}$.

We provide additional graph theory definitions and use a classical graph theory result (Theorem 3.6) to show that for any distribution $\delta$ for $r 1^{r-1}$, the digraph $D(\delta)$ is strongly connected. The indegree $\operatorname{indeg}(v)$ of a vertex $v$ in a digraph $(V, E)$ is the number of distinct vertices $u$ such that $u v \in E$; the outdegree $\operatorname{outdeg}(v)$ of $v$ is the number of distinct vertices $u$ such that $v u \in E$. An Eulerian trail in a connected digraph is a walk which starts and ends at the same vertex, such that every directed edge of the walk occurs exactly once, and every vertex of the walk occurs at least once. A digraph $(V, E)$ is Eulerian if it contains an Eulerian trail. Observe that an Eulerian graph is strongly connected. The next theorem is the directed graph analogue of a classic theorem of graph theory, due to Euler, which provides necessary and sufficient conditions for the existence of an Eulerian trail (see [2, Theorem 2.23]).

Theorem 3.6. If $D$ is a connected digraph such that $\operatorname{indeg}(v)=\operatorname{outdeg}(v)$ for all vertices $v$ of $D$, then $D$ is Eulerian. In particular, $D$ is strongly connected.

Lemma 3.7. Let $\tau=r 1^{r-1}$ and let $\delta$ be a distribution for $\tau$. Then

(1) $D(\delta)$ is connected;

(2) for any $A \in \mathcal{A}_{2 r-1, r}$, we have indeg $(A)=\operatorname{outdeg}(A)$ in $D(\delta)$; and

(3) $D(\delta)$ is strongly connected.

Proof. As the third statement of the lemma is a consequence of Theorem 3.6 and the first two statements of the lemma, we concentrate on proving the first two statements.

Let $A$ and $B$ be distinct sets contained in $\mathcal{A}_{2 r-1, r}$. If $|A \cap B|<r-1$, then it is not difficult to see that there exists a sequence $A=A_{1}, A_{2}, \ldots, A_{k}=B$ of sets in $\mathcal{A}_{2 r-1, r}$, satisfying $\left|A_{i} \cap A_{i+1}\right|=r-1$, for $i=1,2, \ldots, k-1$. In particular, to prove the first statement, it suffices to show that if $|A \cap B|=r-1$, then $A$ and $B$ are in the same connected component of the simple graph associated with $D(\delta)$.

Suppose $|A \cap B|=r-1$. Let $\pi(A, B)$ be the partition of type $\tau$ whose singleton classes are the elements of $A \cap B$. Observe that $\pi(A, B)$ is the unique partition of type $\tau$ which is orthogonal to both $A$ and $B$. Because $\delta$ is a distribution, the partition $\pi(A, B)$ is in the domain of $\delta$. Let $\delta(\pi(A, B))=C$. If $C=B$, then $A B$ is an edge of the digraph $D(\delta)$; if $C=A$, then $B A$ is an edge of $D(\delta)$; if $C$ is distinct from $A$ and $B$, then $A C$ and $B C$ are edges of $D(\delta)$. In particular, $A$ and $B$ are contained in the same connected component of the simple graph associated with $D(\delta)$.

To prove the second statement of the lemma, let $A$ be an $r$-set and $\alpha$ be the unique partition of type $\tau$ such that $\delta(\alpha)=A$. The indegree indeg $(A)$ is the number of $r$-sets 
other than $A$ which are orthogonal to $\alpha$. As observed in Lemma 2.3, the number of $r$-sets orthogonal to $\alpha$ is $r$, thus indeg $(A)$ is $r-1$. The outdegree $\operatorname{outdeg}(A)$ is the number of partitions of type $\tau$, other than $\alpha$, which are orthogonal to $A$. By Lemma 2.3, it follows that outdeg $(A)=r-1$.

Corollary 3.8. If $\tau=r 1^{r-1}$, then the rank and the idempotent rank of $S(\tau)$ are both equal to $\left(\begin{array}{c}2 r-1 \\ r-1\end{array}\right)$.

Remark 3.9. A brief description of Howie and McFadden's method in [7] for proving the existence of a generating set of $S(n, r)$ idempotents for the semigroup $K(n, r)$, for positive integers $n$ and $r$, with $n>r$, is provided below.

For given $n$ and $r$, it is proved in [7] that there exists an orthogonally labelled list $A_{1} \pi_{1} \ldots A_{\left(\begin{array}{c}n \\ r\end{array}\right)} \pi_{\left(\begin{array}{c}n \\ r\end{array}\right)}$. A given orthogonally labelled list determines a set $e_{1}, e_{2}, \ldots, e_{\left(\begin{array}{c}n \\ r\end{array}\right)}$ of idempotents with $\operatorname{ran}\left(e_{i}\right)=A_{i+1}$ and $\operatorname{ker}\left(e_{i}\right)=\pi_{i}$ for $i=1, \ldots,\left(\begin{array}{l}n \\ r\end{array}\right)$. Because the kernels of the idempotents in the set $\left\{e_{i}: i=1, \ldots,\left(\begin{array}{l}n \\ r\end{array}\right)\right\}$ are distinct, this set can be extended to a set $G$ of $S(n, r)$ distinct idempotents with distinct kernels. Thus every $r$-set is represented as a range of some idempotent in $G$, and every partition of weight $r$ is represented as a kernel of a unique idempotent in $G$.

Using an argument similar to that of Lemma 2.2, it is shown that the set $G$ is a generating set for $K(n, r)$. As in the paragraph preceding Proposition 2.4, any generating set for $K(n, r)$ has at least $S(n, r)$ elements; thus, the idempotent rank of $K(n, r)$ is $S(n, r)$. For an implementable algorithm that, on input $n$ and $r$, outputs an orthogonally labelled list, see [13].

For certain partition types $\tau$, in $[\mathbf{8}, \mathbf{1 0}-\mathbf{1 2}]$, the authors generalize the technique of $[\mathbf{7}]$, proving the existence of an orthogonally $\tau$-labelled list and thereby determining the rank and idempotent rank of certain classes of $S(\tau)$ semigroups.

Remark 3.10. We show below that proving the existence of an orthogonally $r 1^{r-1}$ labelled list is equivalent to proving the validity of the Middle Levels Conjecture for $r$.

Suppose

$$
A_{1} \pi_{1} \ldots A_{\left(\begin{array}{c}
2 r-1 \\
r
\end{array}\right)} \pi_{\left(\begin{array}{c}
2 r-1 \\
r
\end{array}\right)}
$$

is an orthogonally $r 1^{r-1}$-labelled list. Recall that $B\left(\pi_{i}\right)$ is the $(r-1)$-set of singleton classes of $\pi_{i}$. Using Lemma 2.3, it follows that $B\left(\pi_{i}\right)=A_{i} \cap A_{i+1}$ and

$$
B\left(\pi_{1}\right), \ldots, B\left(\pi_{\left(\begin{array}{c}
2 r-1 \\
r
\end{array}\right)}\right)
$$

is a sequence of distinct $(r-1)$-sets. Hence existence of an orthogonally $r 1^{r-1}$-labelled list

$$
A_{1} \pi_{1} \ldots A_{\left(\begin{array}{c}
2 r-1 \\
r
\end{array}\right)} \pi_{\left(\begin{array}{c}
2 r-1 \\
r
\end{array}\right)} \text { implies that } A_{1} B\left(\pi_{1}\right) \ldots A_{\left(\begin{array}{c}
2 r-1 \\
r
\end{array}\right)} B\left(\pi_{\left(\begin{array}{c}
2 r-1 \\
r
\end{array}\right)}\right)
$$

is a Hamiltonian cycle of $\mathcal{B}(r)$. Conversely, Hamiltonian cycles of $\mathcal{B}(r)$ give rise to orthogonally $r 1^{r-1}$-labelled lists, as is easy to verify. 
The Middle Levels Conjecture has still another connection with the work here. In a directed graph, a directed Hamiltonian cycle is a directed cycle which passes through every vertex of the graph.

Lemma 3.11. The Middle Levels Conjecture is valid for $r$ if and only if there exists a perfect matching $M$ of $\mathcal{B}(r)$, with associated distribution $\delta_{M}$, such that $D\left(\delta_{M}\right)$ contains a directed Hamiltonian cycle.

Proof. Suppose the Middle Levels Conjecture is valid for $r$ and

$$
\mathcal{C}=A_{1} B_{1} A_{2} B_{2} \ldots A_{\left(\begin{array}{c}
2 r-1 \\
r
\end{array}\right)} B_{\left(\begin{array}{c}
2 r-1 \\
r
\end{array}\right)}
$$

is a Hamiltonian cycle in $\mathcal{B}(r)$. By Lemma $2.3(4)$, the sets $T(\tau)$ and $\mathcal{A}_{2 r-1, r-1}$ are in one-to-one correspondence. For an $(r-1)$-set $B$, let $\theta_{B}$ be the type $r 1^{r-1}$ partition whose singleton classes are the elements of $B$.

Observe that the map $\delta: A_{i} \rightarrow \theta_{B_{i}}$, where $i=1, \ldots,\left(\begin{array}{c}2 r-1 \\ r\end{array}\right)$, is a perfect matching. It follows from the definition of the directed graph $D(\delta)$ that $A_{1} A_{2} \ldots A_{\left(\begin{array}{c}2 r-1 \\ r\end{array}\right)}$ is a directed Hamiltonian cycle of $D(\delta)$.

Conversely, suppose that $M$ is a perfect matching of $\mathcal{B}(r)$, with the distribution $\delta_{M}$, and suppose that $\mathcal{D}=A_{1} A_{2} \ldots A_{\left(\begin{array}{c}2 r-1 \\ r\end{array}\right)}$ is a directed Hamiltonian cycle of $D\left(\delta_{M}\right)$. To complete the proof, it suffices to show that for all distinct $i$ and $j$ in $\left\{1, \ldots,\left(\begin{array}{c}2 r-1 \\ r\end{array}\right)\right\}$, the sets $A_{i} \cap A_{i+1}$ and $A_{j} \cap A_{j+1}$ are distinct $(r-1)$-sets.

To see that $A_{i} \cap A_{i+1}$ consists of $r-1$ elements, note that by the definition of $D\left(\delta_{M}\right)$, there exists a partition $\alpha_{i}$ of type $r 1^{r-1}$ such that $\delta_{M}\left(\alpha_{i}\right)=A_{i+1}$. By Lemma 2.3(3) (b), we have that $\left|A_{i} \cap A_{i+1}\right|=r-1$. Observe that $\alpha_{i}$ is the unique partition of type $r 1^{r-1}$ whose set of singleton classes is $A_{i} \cap A_{i+1}$. Next, suppose that for some $i, j \in$ $\left\{1, \ldots,\left(\begin{array}{c}2 r-1 \\ r\end{array}\right)\right\}$, we have that $A_{i} \cap A_{i+1}=A_{j} \cap A_{j+1}$. In this case, $\alpha_{i}=\alpha_{j}$ and $A_{i+1}=$ $D\left(\delta_{M}\right)\left(\alpha_{i}\right)=D\left(\delta_{M}\right)\left(\alpha_{j}\right)=A_{j+1}$. In particular, we have $i=j$.

For a strongly connected directed graph $D$, for two vertices $u$ and $v$, the distance $d(u, v)$ from a vertex $u$ to to a vertex $v$ is the length of the shortest directed path from $u$ to $v$; the diameter of $D$ is $\max \{d(u, v): u \neq v\}$. For a positive integer $r$, let $c(r)$ be the smallest diameter of all $D(\delta)$ graphs, where $\delta$ is a distribution for $r 1^{r-1}$.

For a minimal generating set $G$ of $S(\tau)$, the terseness of $G$ is the smallest $k$ such that the set of elements of $S(\tau)$ with kernel of type $\tau$ is $G^{k}$. Let $g(r)$ be the minimum among the tersenesses of all minimal generating sets of $S\left(r 1^{r-1}\right)$. It is not difficult to prove that $c(r) \leqslant g(r)$.

Problem 3.12. Determine properties of the functions $c(r)$ and $g(r)$.

\subsection{Idempotent rank of $S(\tau), \tau=d 1^{r-1}, d<r$}

We complete the proof of Theorem 1.1 by proving for $d<r$ and $\tau=d 1^{r-1}$ that the idempotent rank of $S(\tau)$ is $\left(\begin{array}{c}n \\ r-1\end{array}\right)$. 
A partial distribution $\delta$ is said to be strongly connected if the corresponding digraph $D(\delta)$ is strongly connected. A maximal strongly connected subgraph of a digraph is called a strongly connected component.

Lemma 3.13. Let $\tau=d 1^{r-1}, d \geqslant 3$, with $r \geqslant d$. Set $n=d+r-1$. Suppose there exist strongly connected partial distributions

(1) $\delta_{1}: T\left((d-1) 1^{r-1}\right) \rightarrow \mathcal{A}_{n-1, r}$ such that $\operatorname{dom}\left(\delta_{1}\right)$ is a proper subset of $T\left((d-1) 1^{r-1}\right)$, and

(2) $\delta_{2}: T\left(d 1^{r-2}\right) \rightarrow \mathcal{A}_{n-1, r-1}$.

If the domain of $\delta_{1}$ consists of $u$ partitions and the domain of $\delta_{2}$ consists of $v$ partitions, then there exists a strongly connected partial distribution $\delta_{1,2}: T(\tau) \rightarrow \mathcal{A}_{n, r}$ with no more than $u+v+1$ partitions in its domain.

Proof. We show that the partial distributions $\delta_{1}$ and $\delta_{2}$ give rise to partial functions $\tilde{\delta}_{1}$ and $\tilde{\delta}_{2}$ from $T(\tau)$ to $\mathcal{A}_{n, r}$. For each partition $\alpha$ in the domain of $\delta_{1}$ let $\tilde{\alpha}$ be a partition of type $\tau$ obtained from $\alpha$ by adding the element $n$ into its $(d-1)$-class. For each partition $\beta$ in the domain of $\delta_{2}$ let $\tilde{\beta}$ be a partition of type $\tau$ obtained from $\beta$ by adding an extra singleton class $\{n\}$. Let $\tilde{\delta}_{1}$ be a partial function with domain $\left\{\tilde{\alpha}: \alpha \in \operatorname{dom}\left(\delta_{1}\right)\right\}$, defined by $\tilde{\delta}_{1}(\tilde{\alpha})=\delta_{1}(\alpha)$. Since the partial distribution $\delta_{1}$ is surjective, the range of $\tilde{\delta}_{1}$ is the set of all the $r$-subsets of $\mathcal{A}_{n, r}$ that do not contain $n$.

Let $\tilde{\delta}_{2}$ be a partial function with domain $\left\{\tilde{\beta}: \beta \in \operatorname{dom}\left(\delta_{2}\right)\right\}$, defined by $\tilde{\delta}_{2}(\tilde{\beta})=$ $\delta_{2}(\beta) \cup\{n\}$. Since the partial distribution $\delta_{2}$ is surjective, the range of $\tilde{\delta}_{2}$ is the set of all the $r$-subsets of $\mathcal{A}_{n, r}$ that contain $n$.

The domains of $\tilde{\delta}_{1}$ and $\tilde{\delta}_{2}$ are disjoint subsets of $T(\tau)$, while the ranges of $\tilde{\delta}_{1}$ and $\tilde{\delta}_{2}$ are disjoint subsets of $\mathcal{A}_{n, r}$ whose union equals $\mathcal{A}_{n, r}$. Moreover, for $i=1$ or 2 , and any partition $\gamma$ in the domain of $\tilde{\delta}_{i}$, the image $\tilde{\delta}_{i}(\gamma)$ is orthogonal to $\gamma$. Thus, the partial function $\delta_{1,2}^{\prime}$-whose domain is the union of domains of $\tilde{\delta}_{1}$ and $\tilde{\delta}_{2}$, and whose action coincides with that of $\tilde{\delta}_{1}$ and $\tilde{\delta}_{2}$ on their respective domains - is in fact a partial distribution from $T(\tau)$ onto $\mathcal{A}_{n, r}$.

The fact that $\delta_{1}$ and $\delta_{2}$ are both strongly connected implies that the ranges of $\tilde{\delta}_{1}$ and $\tilde{\delta}_{2}$ are strongly connected components of the range of $\delta_{1,2}^{\prime}$. We construct an extension $\delta_{1,2}$ of $\delta_{1,2}^{\prime}$ that is strongly connected.

By hypothesis, there exists a partition $\rho$ of type $(d-1) 1^{r-1}$ that is not contained in the domain of $\delta_{1}$. Hence the partition $\tilde{\rho}$, obtained from $\rho$ by adjoining the element $n$ to its $(d-1)$-class, is not in the domain of $\delta_{1,2}^{\prime}$. Let $A$ be the set of the singleton classes of $\rho$. Then any $r$-set containing $A$ is orthogonal to $\tilde{\rho}$. We extend the domain of $\delta_{1,2}^{\prime}$ to that of $\delta_{1,2}$ by letting $\delta_{1,2}(\tilde{\rho})=A \cup\{n\}$. For any $r$-set $B$ that contains $A$ but not $n$, there is a directed edge from $B$ to $A \cup\{n\}$ in $D\left(\delta_{1,2}\right)$.

To complete the proof, it suffices to show that there exists a directed edge $V U$ in $D\left(\delta_{1,2}\right)$, such that $U$ does not contain $n$ and $V$ contains $n$. Let $\alpha$ be the partition of type $d 1^{r-1}$ satisfying $\delta_{1,2}(\alpha)=U$. By definition of $\delta_{1,2}$, there exists $y$ in $U$ such that $y \neq n$, and $n$ and $y$ are contained in the $d$-class of $\alpha$. Let $W$ be the $(r-1)$-set such that $U=W \cup\{y\}$, and let $V=W \cup\{n\}$. Observe that $\alpha$ is simultaneously orthogonal to both 
$U$ and $V$. Therefore, we have that the equality $\delta_{1,2}(\alpha)=U$ implies there is a directed edge $V U$ in $D\left(\delta_{1,2}\right)$.

The proof of the next proposition completes the proof of Theorem 1.1. For two sets $U$ and $V$ contained in $X_{n}$, let $\Delta(U, V)$ denote their symmetric difference in $X_{n}$.

Proposition 3.14. Let $\tau=d 1^{r-1}$ with $r \geqslant d \geqslant 2$. There exists a strongly connected partial distribution $\delta$ such that the domain of $\delta$ has no more than $\left(\begin{array}{c}n \\ r-1\end{array}\right)-(r-d)$ elements.

Proof. We prove the Proposition by induction on $d$ and $r$. Assume first that $d=2$, so $n=r+1$ and let $A_{i}=X_{n}-\{n-i+1\}$, for $i=1,2, \ldots, n$. We treat all subscripts 'mod $n$ '. For $i=1,2, \ldots, n$, let $\alpha_{i}$ be the partition of $X_{n}$ whose unique non-singleton class is $\triangle\left(A_{i}, A_{i+1}\right)$. Observe that $\alpha_{i}$ is simultaneously orthogonal to $A_{i}$ and $A_{i+1}$. Hence the partial function $\delta$ from $T(\tau)$ onto $\mathcal{A}_{n, r}$, with $\delta\left(\alpha_{i}\right)=A_{i+1}$, is a distribution for $\tau$. The domain of $\delta$ consists of $n=r+1$ partitions. Note that

$$
\left(\begin{array}{c}
n \\
n-1-1
\end{array}\right)-(r-d)=\left(\begin{array}{c}
r+1 \\
2
\end{array}\right)-(r-2)=\left(r^{2}-r+4\right) / 2
$$

As is easily proved, for all $r \geqslant 2$, we have that $\left(r^{2}-r+4\right) / 2$ is an upper bound for $r+1$. Thus, the proposition holds when $d=2$.

Lemmas 3.5 and 3.7 assert the validity of the proposition for the case of $r=d$. We use the cases $d=2$ and $r=d$ as our base steps.

With $d \geqslant 3$ and $r>d$, assume that the proposition is valid for $(d-1) 1^{r-1}$ and for $d 1^{r-2}$. There exist strongly connected partial distributions $\delta_{1}$ and $\delta_{2}$ for partition types $(d-1) 1^{r-1}$ and for $d 1^{r-2}$, respectively, such that the domain of $\delta_{1}$ has no more than $\left(\begin{array}{c}d+r-2 \\ d-1\end{array}\right)-r+d-1$ elements, and the domain of $\delta_{2}$ has no more than $\left(\begin{array}{c}d+r-2 \\ d\end{array}\right)-r+d+1$ elements.

Lemma 3.13 guarantees the existence of a strongly connected partial distribution for $\tau=d 1^{r-1}$ whose domain has no more than

$$
\begin{aligned}
{\left[\left(\begin{array}{c}
d+r-2 \\
d-1
\end{array}\right)-r+d-1\right]+\left[\left(\begin{array}{c}
d+r-2 \\
d
\end{array}\right)-r+d+1\right]+1 } & =\left(\begin{array}{c}
n \\
d
\end{array}\right)-2(r-d)+1 \\
& \leqslant\left(\begin{array}{c}
n \\
r-1
\end{array}\right)-(r-d)
\end{aligned}
$$

elements, using the fact that $r-d \geqslant 1$. This completes the proof by induction.

\section{References}

1. M. Aigner, Lexicographic matching in Boolean algebras, J. Combinat. Theory B 14 (1973), 187-194.

2. G. Chartrand And L. Lesniak, Graphs and digraphs, 2nd edn (Wadsworth and Brooks/Cole Mathematics Series, Monterey, CA, 1986).

3. I. J. DeJter, Two Hamilton cycles in bipartite reflective Kneser graphs, Proceedings of the 1st Japanese conference on graph theory and applications (Hakone, 1986), Discrete Math. 72 (1988), 1-3, 63-70. 
4. D. Duffus, B. SAnds And R. Woodrow, Lexicographic matchings cannot form Hamiltonian cycles, Order 5 (1988), 149-161.

5. J. M. Howie, The subsemigroup generated by the idempotents of a full transformation semigroup, J. Lond. Math. Soc. 41 (1966), 707-716.

6. J. M. HowIE, Idempotent generators in finite full transformation semigroups, Proc. $R$. Soc. Edinb. A 81 (1978), 317-323.

7. J. M. Howie AND R. B. MCFADDEN, Idempotent rank in finite full transformation semigroups, Proc. R. Soc. Edinb. A 114 (1990), 161-167.

8. J. LeHEL AND I. LeVI, Loops with partitions and matchings, Ars Combinatoria 54 (2000), 237-253.

9. I. Levi And R. B. MCFAdDen, $S_{n}$-normal semigroups, Proc. Edinb. Math. Soc. 37 (1994) 471-476.

10. I. LEVI AND S. SEIF, Constant weight Gray codes labeled by partitions with blocks of size at most two, Ars Combinatoria, in press.

11. I. LEvi AND S. SEIF, Constructive techniques for labelling constant weight Gray codes, in preparation.

12. I. LEVI AND S. SEIF, Counting techniques for labelling constant weight Gray codes, in preparation.

13. I. Levi, R. B. McFadden And S. SeIf, Algorithms for labeling monotone Gray codes, Glasgow Math. J., in press.

14. C. D. Savage And P. Winkler, Monotone Gray codes and the middle levels problem, J. Combinat. Theory A 70 (1995), 230-248.

15. S. SEIF, Minimal generating sets for the ideals of the endomorphism semigroup of a finite vector space, in preparation. 